\title{
Brand Experience: Immer noch Neuland in der Markenführung
}

Eine zentrale Frage in Zeiten der digitalen Revolution lautet: Wie können Marken einzigartige Erlebnisse vermitteln? Wissenschaft und Praxis sind sich einig, dass Brand Experience das Potenzial zur Königsdisziplin der Markenführung besitzt. Angesichts von über 3.000 Werbebotschaften, die heutzutage täglich auf die Kunden einströmen, sowie der zunehmenden Austauschbarkeit von Produkten und Dienstleistungen stellen real und medial vermittelte Markenerlebnisse den Schlüssel bei der Pflege und beim Aufbau von starken Marken dar. Im Vergleich zu anderen Disziplinen im Markenmanagement ist jedoch der Wissensstand im Feld der Brand Experience immer noch relativ unterentwickelt. Wie erleben Konsumenten die Marke? Wie lassen sich Markenerlebnisse messen? In welchem Ausmaß und wie beeinflussen Markenerlebnisse das Konsumentenverhalten sowie die Beziehung zur Marke?

Die vorliegende Ausgabe der Marketing Review St. Gallen widmet sich diesen Fragestellungen. Experten aus Wissenschaft und Praxis zeigen auf, welche Rolle einzigartige Markenerlebnisse für den Erfolg einer Marke spielen, und beleuchten, welche Dimensionen und Faktoren der Brand Experience es tatsächlich sind, die den Markenerfolg entscheidend beeinflussen. Im ersten Themenblock stehen zunächst die Erfolgsfaktoren und die Umsetzung der Markenerlebnisse im Vordergrund. Zudem erläutert ein Praxisbeitrag mittels Fallstudien, wie Markenerlebnisse anhand von Kundenbeziehungen gemessen werden. Im zweiten Themenblock finden sich konkrete Hinweise, wie Markenkontaktpunkte gestaltet werden sollen. Während sich ein Beitrag mit der bedürfnisorientierten Bezeichnung und Anordnung von Produktinformationen an On- und Offline-Kontaktpunkten beschäftigt, behandelt ein zweiter Beitrag die Dimensionen des digitalen Markenerlebnisses. Der dritte Themenblock ist dem Thema multisensorischer Erlebniswelten gewidmet.

Wie man Markenkontaktpunkte in der Praxis inspirierend entwickelt, erklärt Ariane Ehrat, Geschäftsführerin der Tourismusorganisation Engadin St. Moritz im Interview. Eine ab-

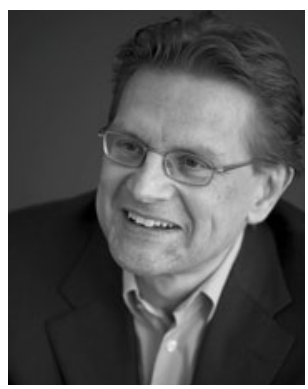

Prof. Dr. Torsten Tomczak Direktor der Forschungsstelle für Customer Insight (FCI) an der Universität St. Gallen.

E-Mail: torsten.tomczak@unisg.ch

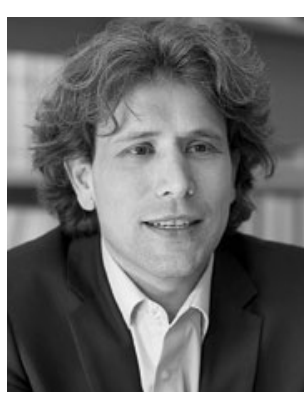

Prof. Dr. Sven Henkel Assistenzprofessor und Vizedirektor der Forschungsstelle für Customer Insight (FCI) an der Universität St. Gallen. E-Mail:sven.henkel@unisg.ch

schliessende Analyse von vier Fallbeispielen illustriert die Bedeutung von Emotionen im Rahmen von Markenerlebnissen.

Wir wünschen eine spannende und inspirierende Lektüre.

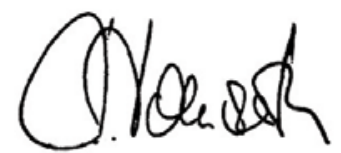

Torsten Tomczak

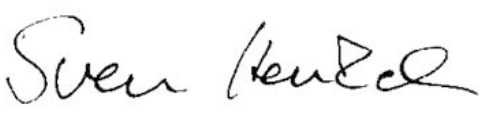

Sven Henkel 